\title{
Apollo EFP: Um Modelo para Cuidado Ubíquo Aplicado à Prevenção de Quedas de Idosos
}

\author{
João Tavares ${ }^{1}$, Bruno Mota ${ }^{1}$, Jorge Barbosa ${ }^{1}$, Adenauer Yamin ${ }^{2}$, Rodrigo Real ${ }^{3}$ \\ ${ }^{1}$ Programa de Pós-Graduação em Computação Aplicada (PPGCA) \\ Universidade do Vale do Rio dos Sinos (UNISINOS) \\ Av. Unisinos, 950 - Bairro Cristo Rei - São Leopoldo, RS, Brasil \\ ${ }^{2}$ Mestrado em Eletrônica e Computação (MEEC) \\ Universidade Católica de Pelotas (UCPel) \\ Rua Félix da Cunha, 412 - Centro - Pelotas, RS, Brasil \\ ${ }^{3}$ Freedom Veículos Elétricos Ltda \\ Rua Conde de Porto Alegre, 155 - Pelotas, RS, Brasil \\ \{joaoer, jbarbosa\} @unisinos.br, motaalvesb@gmail.com \\ adenauer.yamin@ucpel.edu.br, rodrigodfreedom.ind.br
}

\begin{abstract}
According to official data, the accidental fall is the main cause of morbidity due to unintentional external causes to people over 60 years. This article presents a model for ubiquitous care applied to the prevention of falls in the elderly. The classification algorithm adopted is based on relative thresholds, for this purpose, it is proposed the Fall Risk Index (IRQ). The main scientific contribution of this study is the preventive analysis of the risk of falls, considering the profile, the health history and the context of the elderly in intelligent environments. Usability and acceptance evaluations were performed by 2 elderly people in a real setting. The results indicate the approval of $87 \%$ in the perceived ease of use and $100 \%$ in the perceived utility. In addition, we performed tests by simulation of 5 postural states by 10 elderly people with different profiles, resulting in a mean accuracy of the algorithm for prevention and detection of events related to falls of $96 \%$.
\end{abstract}

Resumo. Segundo dados oficiais, a queda acidental é o principal motivo de morbidade por causas externas não intencionais a pessoas com mais de 60 anos. Este artigo apresenta um modelo para cuidado ubíquo aplicado à prevenção de quedas de idosos. O algoritmo de classificação adotado baseiase em limiares relativos, para isso, propõe-se o Índice de Risco de Queda (IRQ). A principal contribuição científica deste trabalho reside na análise preventiva do risco de quedas, considerando o perfil, o histórico de saúde e o contexto do idoso em ambientes inteligentes. Foram realizadas avaliações de usabilidade e aceitação por 2 idosos em um cenário real. Os resultados indicam a aprovação de 87\% na facilidade percebida de uso e 100\% na utilidade percebida. Além disso, foram realizados testes por simulação de 5 estados posturais por 10 idosos com perfis diferentes, resultando em uma acurácia média do algoritmo de prevenção e detecção de eventos relacionados a quedas de $96 \%$. 


\section{Introdução}

Segundo dados da Organização Mundial da Saúde (OMS), em torno de 9\% do total das mortes no mundo, aproximadamente 5 milhões/ano, são resultantes de causas externas [WHO 2019]. No Brasil, entre 2010 e 2015, este índice representou o terceiro principal motivo, atingindo 8,1 \% [Datasus 2019]. A Classificação Estatística Internacional de Doenças e Problemas Relacionados à Saúde, 10 $0^{\mathrm{a}}$ revisão (CID-10) [WHO 2019], em seus capítulos XIX e XX, categoriza as causas externas como intencionais (homicídio por arma de fogo, por exemplo) ou não intencionais ou acidentais (queda, por exemplo).

Anualmente cerca de 40.000 mortes de idosos decorrem de quedas. Para aqueles com mais de 80 anos, a taxa de mortalidade associada a quedas é seis vezes mais alta do que entre 65 e 79 anos, por caírem mais vezes e serem mais frágeis [Cunha e Lourenço 2014]. Complementarmente, as pesquisas apontam para o próprio domicílio como o principal local de ocorrência de morbidade acidental de idosos [Anvisa 2019].

Por outra via, a evolução tecnológica vem proporcionando o aumento do uso de smartphones, dispositivos inteligentes e wearables, consolidando o conceito da Internet das Coisas (IoT). Os Ambientes Inteligentes ( $\mathrm{AmI}$ ) [Sadri 2011] vêm concretizando a visão de computação ubíqua, "invisível" e "onipresente", projetada por Mark Weiser [Weiser 1991]. A convergência destes paradigmas e tecnologias aplicadas às áreas da saúde e acessibilidade, promoveram os campos de pesquisa chamados de Ubiquitous Healthcare [Connelly et al. 2017][Acampora et al. 2013] e Ubiquitous Accessibility [Barbosa 2018][Tavares 2016].

A motivação central deste trabalho reside no gap para o tratamento preventivo de quedas. Verifica-se que a literatura trata geralmente a detecção de quedas [Ramezani et al. 2018][Kau e Chen 2015], com suporte ao pós-queda, normalmente notificando familiares e equipe médica. Adicionalmente, verifica-se que os Sistemas de Detecção de Quedas (SDQ) em geral tratam de modo linear o deslocamento dos usuários, desconsiderando o histórico do idoso e seus fatores intrínsecos, extrínsecos e ambientais. Por isso, propomos neste trabalho o Índice de Risco de Queda (IRQ), como um indicador amplo e dinâmico.

A principal contribuição científica do Apollo Elder Fall Prevention (EFP) reside na análise preventiva do risco de quedas, considerando o perfil [Dey 2001], o histórico de saúde e o contexto [Satyanarayanan 2001] do idoso em ambientes inteligentes, para além da detecção. O Apollo EFP permite ainda o acompanhamento dos idosos por seus familiares através de alertas de risco e notificações, bem como um relatório de gestão de riscos. Além disso, o Apollo EFP possibilita ao idoso solicitar apoio emergencial via chamada de voz e mensagem de SMS/E-mail automáticos, com dados de sua localização (via GPS). Assim, espera-se contribuir para a redução do índice de morbimortalidade por conta desta causa.

O texto está organizado em 7 seções, onde a Seção 2 descreve e compara os trabalhos relacionados. A Seção 3 apresenta o modelo Apollo EFP, sua arquitetura e módulos. Na Seção 4 apresentam-se os protótipos desenvolvidos. Nas Seções 5 e 6 contemplam-se os experimentos realizados e os resultados obtidos. Finalmente na Seção 7 são expostas as conclusões e trabalhos futuros. 


\section{Trabalhos Relacionados}

Essa seção apresenta trabalhos relacionados que abordam a análise da queda de idosos utilizando-se computação ubíqua.

Faisal et al. [2018] apresentam uma abordagem para o monitoramento de quedas baseada em aprendizado de máquina supervisionado. Os autores propõem alguns cenários da vida cotidiana $(A D L s)$ em que os usuários utilizam wearables para coleta de dados de aceleração e giroscópio. Os resultados indicam acurácia de 99,45\% do algoritmo de detecção.

Ramezani et al. [2018] desenvolveram um sistema de detecção de quedas utilizando a fusão de dados de Wi-Fi CSI e acelerômetro. Os autores propõem um sistema "passivo e não invasivo", baseando-se na vibração do chão gerada pela queda do idoso em um quarto. Os resultados indicam acurácia média de 95\% de detecção.

Kau e Chen [2015] propõem um sistema para detecção de quedas e suporte a resgate de emergência. $O$ trabalho implementa a análise do ângulo da bússola eletrônica e do acelerômetro do smartphone como dados de entrada. Utiliza também a localização de GPS para apoiar no suporte pós-queda dos usuários. O algoritmo de classificação utilizado é composto de diversos métodos: State Machine/Cascade Classifier, STFT, SVM, HPF e Hear DWT. Os resultados indicam acurácia acima de 92\%.

A Tabela 1 sumariza o comparativo entre o Apollo EFP e os trabalhos relacionados.

Tabela 1. Comparativo entre os trabalhos relacionados

\begin{tabular}{|c|c|c|c|c|}
\hline Quesito & $\begin{array}{c}\text { Faisal et al. } \\
2018 \\
\end{array}$ & $\begin{array}{c}\text { Ramezani et al. } \\
2018\end{array}$ & $\begin{array}{c}\text { Kau e Chen } \\
2015 \\
\end{array}$ & $\begin{array}{c}\text { Apollo } \\
\text { EFP }\end{array}$ \\
\hline Sistema de apoio à tomada de decisão & Não & Não & Não & Sim \\
\hline Variáveis do ambiente & Não & Não & Não & Sim \\
\hline Histórico de saúde & Não & Não & Não & Sim \\
\hline Prevenção de quedas & Sim & Não & Não & Sim \\
\hline SOS & Não & Não & Não & Sim \\
\hline Chamada serviço de emergência & Não & Não & Sim & Sim \\
\hline Notificação a familiares / cuidadores & Não & Não & Sim & Sim \\
\hline
\end{tabular}

A partir da análise comparativa entre o modelo Apollo EFP e os trabalhos relacionados constata-se que sua principal contribuição científica está na prevenção da queda, através do monitoramento contínuo baseado no IRQ considerando-se dados do usuário, do ambiente e de seu histórico de saúde. Ou seja, desequilíbrios parciais ou progressivos, que em geral ocorrem antes de uma queda, são detectados, notificados e registrados pelo Apollo EFP. Além do acompanhamento via relatório de gestão do risco de quedas. Alguns diferenciais ainda podem ser destacados: (1) considera o histórico de saúde idoso - fatores intrínsecos e extrínsecos; (2) considera múltiplos sensores (acelerômetro, giroscópio, luminosidade, temperatura); (3) implementa a adaptabilidade do sistema de detecção dinamicamente - IRQ dinâmico; (4) possibilita o acompanhamento dos movimentos do idoso, enviando notificações quando necessário, agregando o "acompanhamento" e "assistência"; (5) age proativamente, ativando/desativando recursos do ambiente (iluminação). 


\section{Modelo Apollo EFP}

A Figura 1 mostra a arquitetura do modelo Apollo EFP, a qual é composta por dez componentes. Perfil do Usuário (MPU), Ambiente Inteligente (MAI), Mapeamento de Risco (MMR), Análise de Risco de Queda (ARQ), Gestão de Risco de Queda (GRQ) e Configuração (MC), são orientados a Web Services. O Assistente Pessoal do Idoso (API), Assistente de Ambiente Inteligente (AAI) e Assistente de Comunicação (AC) são agentes de software. O Web Portal direciona-se para a administração dos registros e configurações gerais que são persistidos no Banco de Dados (BD).

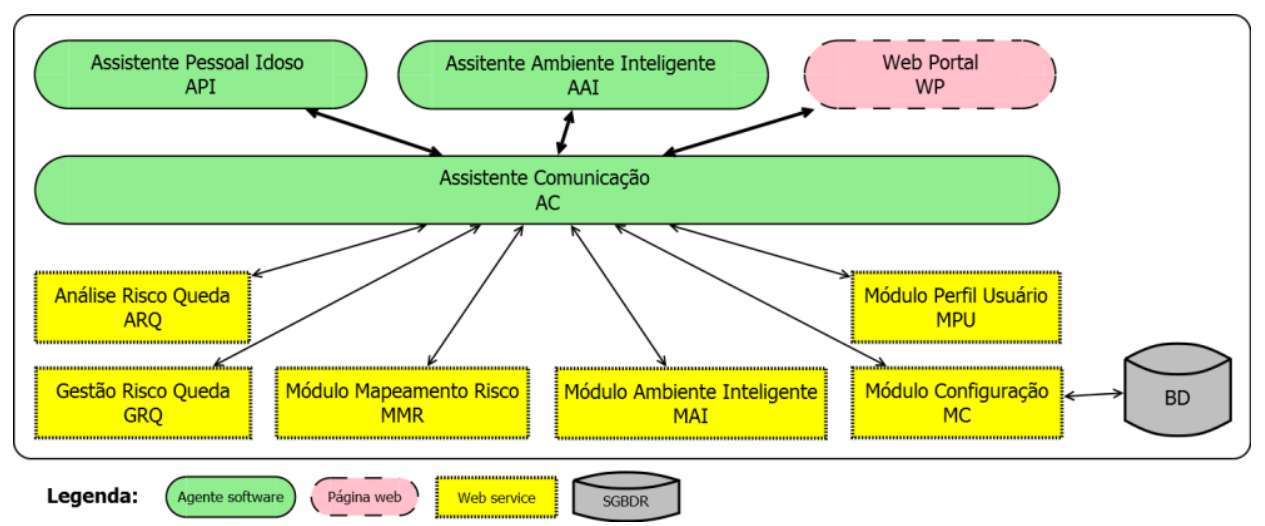

Figura 1. Arquitetura do Modelo Apollo EFP

O API é um assistente pessoal focado no acompanhamento dos movimentos do idoso. Por meio desse agente, ocorre a comunicação, via AC, com o MPU para obtenção de informações do perfil do usuário e com o ARQ para envio dos dados de deslocamento e localização, retornando o status de risco de queda, que podem gerar notificações. O AAI responsabiliza-se por coletar os dados dos sensores e atuadores do ambiente inteligente e comunicar-se via AC com o MAI e MMR, para fornecer subsídios ao ARQ quanto às variáveis ambientais a serem processadas para o cálculo do Índice de Risco de Queda (IRQ).

O módulo ARQ responsabiliza-se por analisar os dados de deslocamento do usuário e do ambiente para prever e detectar as quedas, aplicando o algoritmo de classificação por limiares relativos, considerando-se o IRQ, que é calculado continuamente em base a 4 fatores ponderáveis: (1) Fator do Usuário (FU); (2) Fator do Ambiente (FA); (3) Fator Intrínseco (FI) - 27 variáveis mapeadas, tais como doenças neurológicas, por exemplo; (4) Fator Extrínseco (FE) - 22 variáveis mapeadas - tais como mobilidade reduzida, por exemplo. A literatura em geral considera o valor limiar de aceleração 6 (6G) como referência para detecção de queda, enquanto ignora o risco de queda e alertas. Este trabalho considera o IRQ como fator de sensibilidade do método de detecção, pois idosos possuem graus diferentes de autonomia. Logo, quanto maior o IRQ (para casos de idosos com mais riscos devido ao perfil e acúmulo de fatores intrínsecos e extrínsecos), menor a aceleração linear necessária para alerta, notificação e deteç̧ão de quedas.

O módulo GRQ realiza a compilação diária dos eventos ocorridos com o idoso e os envia por e-mail para os contatos de familiares registrados no BD. São informados os dados do idoso, seu histórico de movimentação por hora, indicando de forma gráfica se o idoso esteve parado ou em movimento, as ocorrências de alertas, feedbacks, quedas, 
SOS, etc. Apresentando inclusive o contexto e o local dos eventos. Existem 7 eventos tratados pelo Apollo EFP: (1) Alerta; (2) Risco de queda; (3) Queda; (4) Feedback; (5) SOS; (6) Luz ligada; (7) Luz desligada. Finalmente, o MC responsabiliza-se pelas configurações gerais de perfis, notificações e demais chamadas de acesso ao BD. O Web Portal serve de interface para o idoso, familiares, cuidadores e médicos administrarem as informações do BD.

\section{Protótipos}

Um protótipo do modelo Apollo EFP foi desenvolvido, subdivido em dois apps e web services. Os apps Apollo EFP Motion e Env foram desenvolvidos em Android e instalados em smartphones Motorola Z3, utilizando-se os sensores embarcados. Motion: acelerômetro (frequência de coleta de $50 \mathrm{~Hz}$, com intervalo de coleta de 1,5 segundos, resultando em um array de 75 amostras por pacote), giroscópio, sensor de luminosidade e GPS. Env: Luminosidade e temperatura do local (utilizou-se a API Yahoo Weather).

O Motion considera o contexto de localização indoor ou outdoor obtendo-se pela API do Android a interface de rede utilizada: WiFi, indoor ou 4G, outdoor. Este app, atua como um assistente pessoal emitindo alertas ao idoso quando situações críticas são identificadas, por exemplo, desequilíbrios ou variações no ritmo do caminhar, requisitando feedbacks via pressionamento do botão físico de volume do smartphone, pois este fica posicionado lateralmente, mais acessível e possui tamanho maior. O idoso deve responder à notificação ou alerta em até 60 segundos, pressionando o botão do volume, para que a notificação a familiares ou chamada de emergência não sejam acionados.

O Env, instalado no quarto ou sala da residência do idoso. Caso o Env identifique baixa luminosidade combinada com o deslocamento indoor do idoso, a iluminação de emergência (LED flash auxiliar) é acionada automaticamente, visando mitigar o risco da baixa luminosidade, mantendo-se ligada até que a iluminação do ambiente normalize.

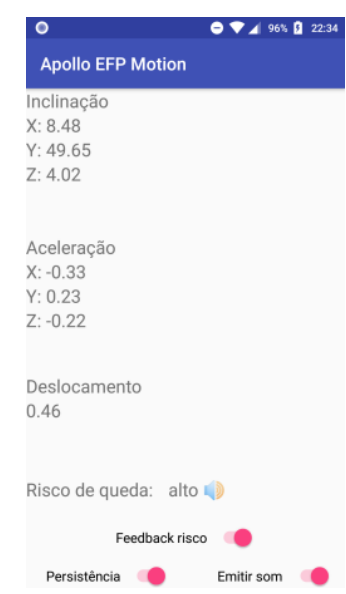

(a)

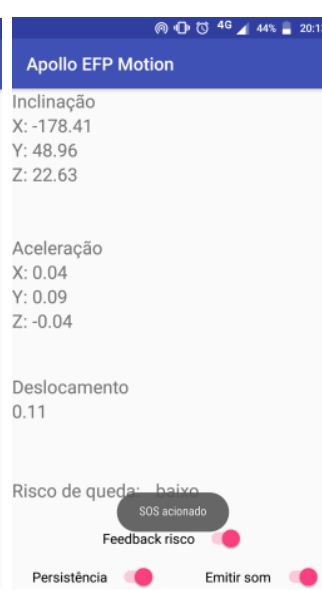

(b)

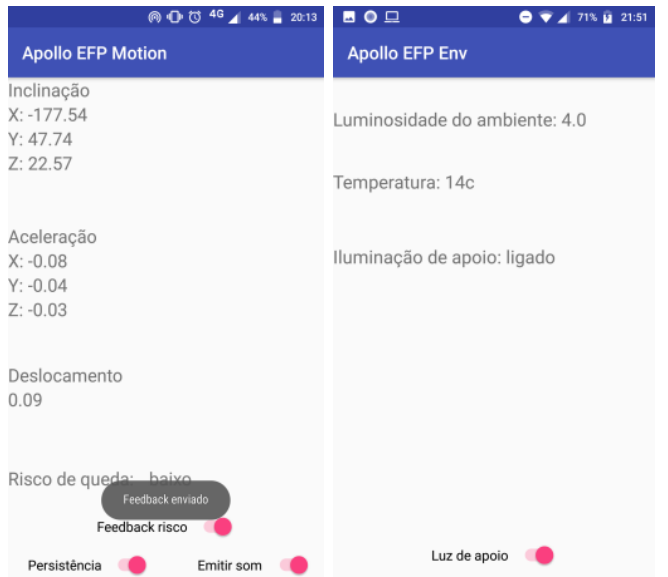

(c)

(d)

Figura 2. Telas dos apps Apollo EFP (a) Motion, (b) Motion - SOS, (c) Motion Feedback e (d) Env 
A Figura 2a apresenta a tela principal do Apollo Motion, que exibe os dados de inclinação por eixo (X, Y ou $\mathrm{Z})$, aceleração, o indicador de deslocamento $\left(\sqrt{a c c X^{2}+a c c Y^{2}+a c c Z^{2}}\right.$ ), o resultado da análise do risco de queda e os switches de configuração. A Figura $2 \mathrm{~b}$ exibe a tela do Apollo EFP Motion logo após o idoso pressionar o botão físico do volume do smartphone por 3 segundos, acionando os contatos de emergência. A Figura 2c apresenta a tela do Apollo EFP Motion com a notificação de feedback enviada ao idoso, com vibração, após sua interação sobre um alerta de risco de queda. A Figura 2d apresenta a tela do Apollo EFP Motion que exibe os dados ambientais da residência do idoso: temperatura e luminosidade.

O Apollo EFP Server foi desenvolvido em Java, disponibilizando-se acesso via Web Service REST. O SGBD utilizado foi o PostgreSQL. O hardware do servidor foi um laptop Dell 7560, com 8GB DDR4 RAM, CPU i5-7200U e SSD 128GB M2, com sistema operacional Windows 10, servidor de aplicações GlassFish.

\section{Avaliação baseada em cenários}

A comunidade científica tem utilizado cenários para avaliação de aplicações ubíquas e sensíveis ao contexto. Partindo dessa estratégia, foi criado um cenário para avaliação do Apollo EFP. Foram realizadas coletas de dados de temperatura e luminosidade de uma sala do Laboratório de Computação Móvel e Ubíqua (Mobilab) que simula o quarto de um idoso. O Motion foi utilizado por um usuário não idoso, portando um smartphone Moto Z3, movimentando-se no quarto simulado, perfazendo os 5 estados posturais, na ordem, que estão elencados na Tabela 2.

O objetivo deste cenário foi avaliar a acurácia do módulo ARQ quanto à prevenção e detecção de quedas, evitando-se a exposição de idosos aos riscos de experimentos reais. Optou-se pela simulação com a maior proximidade possível do cenário real, gerando-se o dataset base para os experimentos simulados. Foram realizadas 10 repetições de coleta de cada um dos 5 movimentos. Após a filtragem, armazenou-se no BD a coleta com menor ruído para cada um dos 5 cenários. A seguir, foram registrados no BD do Apollo EFP Server 10 idosos com perfis variados, com IRQs progressivos, desde a idade de 60 até 85 anos, com diferentes níveis de autonomia (fatores intrínsecos e extrínsecos variados).

Foram realizadas 50 simulações, combinando-se os 10 perfis com os 5 cenários apresentados. Todos os eventos gerados ficaram armazenados na tabela evento do BD. Com base nos dados obtidos nos experimentos, que podem ser visualizados na Tabela 2, aplicou-se a métrica de acurácia $(\mathrm{VP}+\mathrm{VN} / \mathrm{N}$, onde $\mathrm{N}=\mathrm{VP}+\mathrm{FP}+\mathrm{VN}+\mathrm{FN})$, para avaliar o desempenho do modelo na prevenção e detecção de quedas.

Tabela 2: Indicadores dos experimentos realizados por estado postural

\begin{tabular}{|c|c|c|c|c|}
\hline Estado postural & $\begin{array}{c}\text { VP } \\
\text { Verdadeiro } \\
\text { Positivo }\end{array}$ & $\begin{array}{c}\text { FP } \\
\text { Falso } \\
\text { Positivo }\end{array}$ & $\begin{array}{c}\text { FN } \\
\text { Falso } \\
\text { Negativo }\end{array}$ & $\begin{array}{c}\text { VN } \\
\text { Verdadeiro } \\
\text { Negativo }\end{array}$ \\
\hline Parado (EP1) & 10 & 0 & 0 & 0 \\
\hline Caminhando em ritmo normal (EP2) & 10 & 0 & 0 & 0 \\
\hline Desequilíbrio frontal sem queda (EP3) & 9 & 1 & 0 & 0 \\
\hline Caminhando em ritmo rápido (EP4) & 10 & 0 & 0 & 0 \\
\hline Queda frontal (EP5) & 9 & 0 & 1 & 0 \\
\hline Total & 48 & 1 & 1 & 0 \\
\hline
\end{tabular}


A partir dos resultados apresentados na Figura 3, observa-se que os estados EP1, EP2 e EP4 indicam 100\% de acurácia, pois o módulo ARQ não indicou nenhum evento de risco de queda ou queda efetiva, conforme era esperado, pois estes três estados posturais não sofreram variações bruscas no indicador de deslocamento - aceleração média para todos os 10 idosos não ultrapassou $2 \mathrm{G}$ - tampouco no indicador de inclinação do corpo do idoso - para todos os 10 casos não ultrapassou $20^{\circ}$ no eixo $\mathrm{Y}$. Mesmo nos casos em que o IRQ do idoso foi o mais elevado - próximo de 1 - não foi apontado alerta, risco ou queda pelo ARQ.

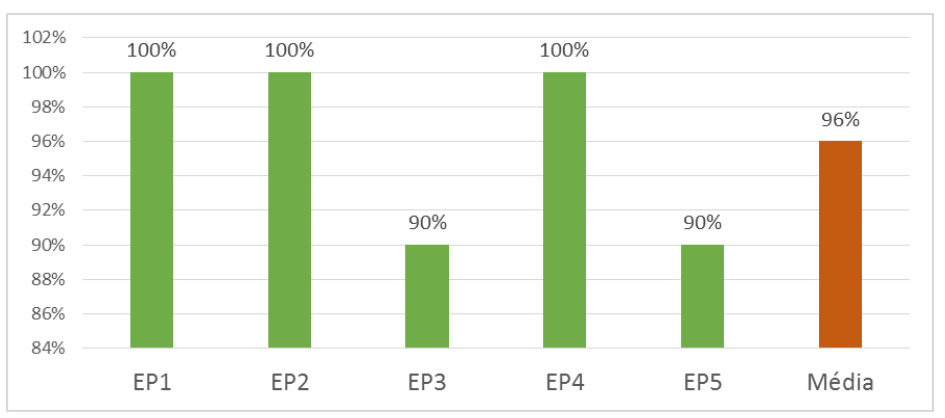

Figura 3: Resultados dos experimentos por estado postural e média global

Para os estados EP3 e EP5 obteve-se 90\% de acurácia. Para o EP3 foi gerado um falso alerta de risco de queda, para um idoso com IRQ alto, próximo de 1, que na prática não deveria ter sido gerado. Analisando-se os registros constatou-se que o mesmo foi gerado por estar muito próximo do limiar de disparo do evento de risco de queda. Entretanto, se fosse um caso real, não haveria danos ao idoso, apenas um falso alerta que poderia ser desativado pela ação do próprio idoso na interface do Motion.

Para o EP5 não foi identificada uma queda que deveria ter sido reportada pelo ARQ. Inversamente ao caso EP3, identificou-se que este falso negativo ocorreu devido ao baixo IRQ do idoso, de modo que o ARQ não o considerou como um caso de queda, mas de risco de queda apenas. Caso fosse um caso real e o idoso não interagisse com o feedback ao Motion, seriam acionados automaticamente os contatos de emergência. Haveria ainda a possibilidade do idoso acionar o SOS.

Finalmente, a acurácia média geral de 96\% indica que o Apollo EFP atua de modo a prevenir a queda de idosos, gerando alertas e notificações para casos de risco. Além disso, o contato emergencial automático ou via $S O S$, contribuem para os casos de detecção de quedas. O histórico de eventos que também pode auxiliar os familiares e responsáveis no acompanhamento do idoso.

\section{Avaliação de usabilidade}

Para fazer a avaliação de aceitação do modelo Apollo EFP, foi elaborado um questionário com base nos conceitos do modelo de aceitação de tecnologia Technology Acceptance Model (TAM) [Yoon e Kim 2007].

As avaliações contaram com a participação voluntária de dois idosos, com 62 anos (masculino) e 61 anos (feminino), sem doenças crônicas ou limitações em sua autonomia. O procedimento adotado para o recrutamento de participantes ocorreu por meio de convite de acordo com a conveniência do experimento, não havendo embasamento estatístico para a quantidade considerada de usuários idosos. 
De modo controlado, para garantir a segurança dos envolvidos, realizou-se a fixação do smartphone Moto Z3 junto ao peito dos usuários, com uma cinta elástica. Após a fixação e explicação geral sobre o experimento, solicitou-se que os mesmos simulassem, caminhando, o movimento de queda frontal, inclinando a parte superior do corpo para frente em um ângulo mínimo de $45^{\circ}$, com aceleração superior a $1 \mathrm{G}$. $\mathrm{O}$ Motion foi programado para emitir áudio de alerta de risco para esta configuração, demandando feedback do usuário (pressionar botão do volume) em até 60 segundos. Após o usuário pressionar o botão de feedback, uma vibração de 2 segundos era sinalizada, para confirmação do comando. Foram solicitados ainda testes do comando de $S O S$ (pressionar o botão volume por 3 segundos) e de uso do Env em uma sala com luminosidade alta e sem iluminação.

Após testarem a aplicação, os mesmos responderam ao questionário proposto, conforme visualiza-se na Tabela 3, que utilizou a escala de Likert, na qual os participantes especificam seu nível de concordância com cada item.

Tabela 3. Itens do questionário sobre facilidade de uso e utilidade percebida

\begin{tabular}{|c|l|}
\hline Item & Facilidade percebida de uso \\
\hline 1 & As informações sobre os riscos de queda são apresentadas de forma clara, sendo de fácil compreensão. \\
\hline 2 & É fácil a compreensão de como usar os recursos prevenção e detecção de quedas. \\
\hline 3 & Não é necessário muito esforço para configurar e usar o Apollo EFP. \\
\hline 4 & O Apollo EFP facilita a prevenção e detecção de quedas. \\
\hline 5 & As notificações sobre riscos de queda foram úteis para a minha necessidade. \\
\hline 6 & O Apollo EFP facilita a prevenção e detecção de quedas em ambientes inteligentes. \\
\hline 7 & O Apollo EFP seria útil para oferecer suporte aos idosos. \\
\hline 8 & O SOS é útil para apoiar o idoso em casos de emergência. \\
\hline
\end{tabular}

Ao analisar os itens que compõem a parte do questionário referente à facilidade percebida de uso (itens 1-4 da Tabela 3), observa-se, conforme está representado na Figura 4a que, $87 \%$ aprovaram e consideraram o Apollo EFP sem dificuldades encontradas para o manuseio. Desse percentual alcançado, 25\% disseram que concordavam totalmente e $62 \%$ concordavam parcialmente. Apenas $13 \%$ respondeu que não concorda nem discorda que é necessário muito esforço para configurar e usar o app. Não houveram respostas quanto ao discordo parcialmente ou discordo totalmente.

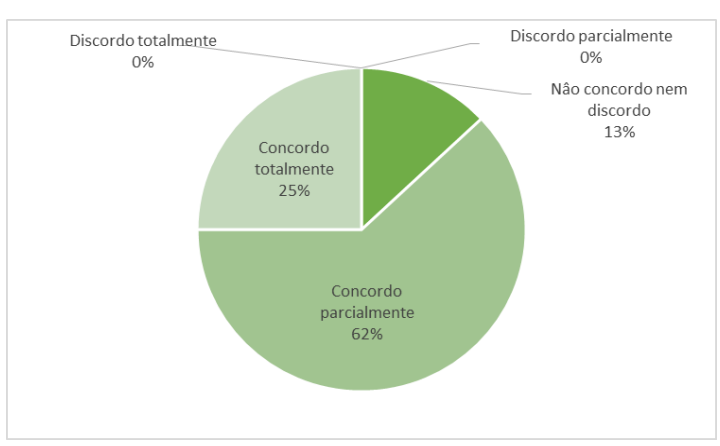

(a)

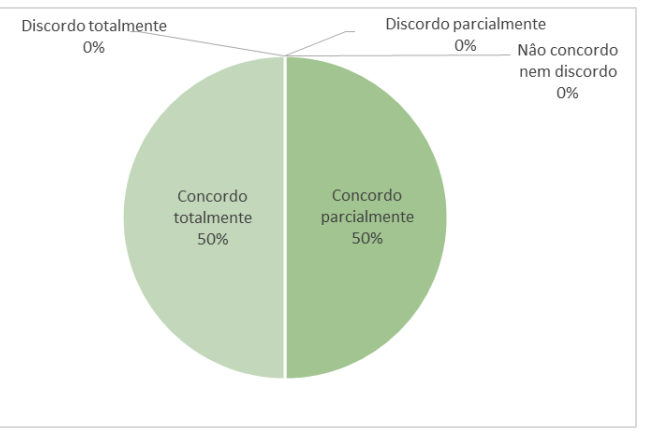

(b)

Figura 4: Resultados da avaliação de usabilidade: (a) Facilidade percebida de uso e (b) Utilidade percebida

Em relação à utilidade percebida (itens 5-8 da Tabela 3), visualiza-se na Figura $4 \mathrm{~b}$ os seguintes percentuais: $50 \%$ de concordo totalmente e $50 \%$ concordo parcialmente, totalizando, assim, $100 \%$ de aprovação quanto à sua utilidade. Não houveram respostas quanto ao discordo parcialmente, discordo totalmente ou não discordo nem concordo. 
Logo, entende-se que o Apollo EFP teve uma boa avaliação no requisito de aprovação quanto à utilidade percebida.

Finalmente, coletarem-se as opiniões de modo livre - para uma avaliação qualitativa de uso do app Apollo EFP Motion e Env - quanto à interface baseada em áudio, vibração e feedbacks para os experimentos realizados. Destaca-se que a principal dificuldade registrada se referiu ao acesso ao botão do volume lateralmente ao peito. Relataram preferir um botão mais acessível. Entretanto ambos os usuários registraram que a solução é relevante e importante para auxiliar na prevenção de quedas e a função de $S O S$ pode ser útil em situações de emergência. O Env foi elogiado como recurso importante no auxílio de iluminação auxiliar automática e as notificações por áudio e vibração são muito válidas.

\section{Conclusões}

Este artigo apresentou o modelo Apollo EFP. Analisaram-se três trabalhos relacionados e constatou-se que o Apollo EFP agrega como principal contribuição científica a prevenção de quedas de idosos, considerando-se o perfil, o histórico de saúde e o seu contexto em um ambiente inteligente. Os protótipos implementados, Env e Motion, permitiram a realização dos testes e avaliações.

A avaliação de usabilidade com 2 idosos obteve como resultados $87 \%$ e 100\%, respectivamente, para a facilidade e utilidade percebida de uso. A avaliação simulada com 10 idosos perfazendo 5 estados posturais diferentes, resultou em $96 \%$ de acurácia média do algoritmo de prevenção e detecção de quedas.

Finalmente destacam-se como possíveis trabalhos futuros: (1) implementar um algoritmo de predição de quedas baseando-se nos padrões históricos; (2) aprimorar o algoritmo de detecção para um método híbrido; (3) utilizar wearables com mais indicadores físiológicos (batimentos cardíacos, respiração, etc); (4) mapear e monitorar fatores de risco ambiental adicionais - piso escorregadio, por exemplo.

\section{Agradecimentos}

Os autores agradecem à FAPERGS, à CAPES - Código de Financ. 001, ao CNPq, à Freedom e à Unisinos pelo apoio ao desenvolvimento desse trabalho. Os autores reconhecem especialmente o apoio do PPGCA e do Mobilab da Unisinos.

\section{Referências}

Acampora, G.; Cook, D.; Rashidi, P. (2013). A Survey on Ambient Intelligence in Healthcare, Proceedings of the IEEE, Vol. 101, Issue 12, Dezembro, 2013. Disponível em: https://doi.org/10.1109/JPROC.2013.2262913.

Anvisa (2019). Protocolo para prevenção de quedas. Disponível em: http://www20.anvisa.gov.br/segurancadopaciente/index.php/publicacoes/item/preven cao-de-quedas. Acesso em 15 março 2019.

Barbosa, J.; Tavares, J.; Cardoso, I.; Mota, B.; Martini, B. (2018). TrailCare: an Indoor and Outdoor Context-aware System to Assist Wheelchair Users. International Journal of Human-Computer Studies, vol. 116, pp. 1-14, 2018 . doi: https://doi.org/10.1016/j.ijhcs.2018.04.001 
Connely, K.; Mayora, O.; Favela, J. (2017). The Future of Pervasive Health. IEEE Pervasive Computing, No. 16, Issue 1, pp. 16-20. Disponível em: https://doi.org/10.1109/MPRV.2017.17.

Cunha, A. e Lourenço, R. (2014). Quedas em idoso: prevalências e fatores associados. In: Revista Hospital Universitário Pedro Ernesto, Vol. 12, № 2 - Envelhecimento e quedas. Disponível em: http://revista.hupe.uerj.br/detalhe_artigo.asp?id=468.

Datasus (2019). Estatísticas de saúde no Brasil, Disponível em: http://tabnet.datasus.gov.br/cgi/tabcgi.exe?sim/cnv/obt10uf.def. Acesso em 15 março 2019.

Dey, Anind K. (2001). Understanding and Using Context, In: Personal and Ubiquitous Computing, Vol. 5, pp. 4-7, Fevereiro, 2001. doi: http://dx.doi.org/10.1007/s007790170019.

Faisal, H.; Muhammad, E.; Muhammad A.; Asra, K. (2018). Elderly Assistance Using Wearable Sensors by Detecting Fall and Recognizing Fall Patterns. ACM International Joint Conference and 2018 International Symposium on Pervasive and Ubiquitous Computing and Wearable Computers (UbiComp '18). ACM, New York, NY, USA, 770-777. DOI: https://doi.org/10.1145/3267305.3274129

Kau, Lih-Jen e Chen, Chih-Sheng (2015). A Smart Phone-Based Pocket Fall Accident Detection, Positioning, and Rescue System, In: Journal of Biomedical and Health Informatics, Vol. 19, N. 1, Janeiro, 2015.

Ramezani, R.; Xiao, Y.; Naeim, A. (2018). Sensing-Fi: Wi-Fi CSI and accelerometer fusion system for fall detection. IEEE EMBS International Conference on Biomedical \& Health Informatics (BHI), Las Vegas, NV, 2018, pp. 402-405. doi: 10.1109/BHI.2018.8333453.

Sadri, Fariba (2011). Ambient Intelligence: A Survey, ACM Computing Survey, Vol. 43, Issue 4, 2011. doi: http://doi.org/10.1145/1978802.1978815.

Satyanarayanan, M. (2001). Pervasive Computing: Vision and Challenges, In: IEEE Personal Communications. pp. 10-17. 2001. 8 (4), pp. 10-17. Disponível em: http://dx.doi.org/10.1109/98.943998.

Tavares, J.; Barbosa, J.; Cardoso, I.; Costa, C.; Yamin, A.; Real, R. (2016). Hefestos: an intelligent system applied to ubiquitous accessibility. Universal Access in the Information Society, pp. 1-19, 2016. doi: http://dx.doi.org/10.1007/s10209-0150423-2.

Weiser, M. (1991). The computer for the 21st century. In Scientific American, pp. 94104, Setembro, 1991. 265 (3), pp. 94-104. doi: http://dx.doi.org/10.1145/329124.329126.

WHO (2019). World Health Organization. Disponível em: http://www.who.int/. Acesso em: 15 março 2019.

Yoon, C. e Kim, S. (2007): Convenience and TAM in a ubiquitous computing environment: the case of wireless LAN. Electron. Commer. Res. Appl. 6(1), 102-112 (2007). doi:10.1016/j.elerap.2006.06.009. 